\title{
Ecografía para la evaluación inicial de pacientes con sospecha de hidatidosis hepática
}

\author{
Ultrasound for the initial evaluation of patients with suspected hepatic hydatidosis
}

María Candelaria Ligo ${ }^{a}$ (D), Morena Montalvo ${ }^{a}(\mathbb{D})$, María Victoria Salgado $b, c, d(D)$

\begin{abstract}
Resumen
La hidatidosis o equinococosis quística es una infección endémica en varias regiones de Argentina. Su forma más frecuente, la hidatidosis hepática, suele ser asintomática por largos periodos de tiempo y, de presentar síntomas, los mismos suelen ser solapados, inespecíficos y crónicos. Por este motivo, los métodos complementarios de diagnóstico resultan indispensables en la valoración de estos pacientes. Entre ellos, la ecografía continúa siendo el método de elección con el que iniciar la evaluación ante la sospecha de hidatidosis hepática; otros métodos, como la serología, la tomografía computada o la resonancia magnética, pueden resultar de utilidad en casos específicos y siempre contextualizados por la clínica, epidemiología, y los hallazgos ecográficos.
\end{abstract}

\begin{abstract}
Hydatidosis or cystic echinococcosis is an endemic infection in several regions of Argentina. Its most frequent form, hepatic hydatidosis, is usually asymptomatic for long periods of time and, if symptoms occur, they are usually overlapping, nonspecific and chronic. For this reason, complementary diagnostic methods are essential in the evaluation of these patients. Among them, ultrasound remains the method of choice to initiate the evaluation of suspected hepatic hydatidosis; other methods, such as serology, computed tomography or magnetic resonance imaging, may be useful in specific cases and always contextualised by the clinical, epidemiology and ultrasound findings.
\end{abstract}

Palabras clave: Equinococosis, Diagnóstico, Ultrasonografía. Keywords: Echinococcosis, Diagnosis, Ultrasonography.

Ligo MC, Montalvo M, Salgado MV. Ecografía para la evaluación inicial de pacientes con sospecha de hidatidosis hepática. Evid Actual Pract Ambul. 2021;24(3):e002133. Available from: https://dx.doi.org/10.51987/EVIDENCIA.V24I4.6962.

\section{Escenario clínico}

Asiste a la consulta de una médica de familia de una zona rural de Santa Cruz un hombre de 42 años por presentar dolor abdominal sordo en hipocondrio derecho de cuatro años de evolución, de intensidad leve, que no lo despierta por la noche. Niega descenso de peso o cambios en el hábito evacuatorio, y refiere no presentar antecedentes patológicos ni tomar medicación de manera habitual. El paciente trabaja desde hace 25 años como peón rural en una estancia cercana, en la que vive. Al examen físico, la médica constata la presencia de una masa palpable en el abdomen superior derecho. Sabiendo que la región en que se encuentra es endémica de hidatidosis, y considerando el ámbito laboral del paciente, la médica se plantea cuál debería ser el estudio complementario de elección con el que evaluar este probable diagnóstico.

\section{Pregunta que generó el caso}

En habitantes de regiones endémicas de hidatidosis que se presentan con cuadros de dolor abdominal en hipocondrio derecho y masa palpable, y ante la sospecha de hidatidosis hepática: ¿es la ecografía el método de elección para realizar el diagnóstico de esta condición, en comparación con la serología u otros métodos por imágenes?

\section{Estrategia de búsqueda}

Se realizó una búsqueda en Google Scholar y Pubmed empleando como palabras claves 'Echinococcosis' y 'Diagnostic Imaging' or 'Diagnostic Techniques and Procedures'. Asimismo, se indagó la bibliografía citada en los artículos originalmente identificados mediante la estrategia de búsqueda descripta. De manera adicional, se revisaron las guías de práctica clínica sobre hidatidosis del Ministerio de Salud de la Nación de Argentina ${ }^{1}$, y el consenso de expertos para el diagnóstico y tratamiento de la equinococosis quística y alveolar en humanos de la Organización Mundial de la Salud (OMS) ${ }^{2}$, y sus listados de referencias.

Se seleccionaron cuatro estudios primarios ${ }^{3-6}$ por considerarlos los más relevantes para responder a la pregunta planteada.

\section{Algunos datos sobre el diagnóstico de Hidatidosis hepática}

La hidatidosis o equinococosis quística es una zoonosis causada por el estadio larvario del cestode Echinococcus Granulosus. Es un ejemplo de la infección humana accidental, ya que, si bien el microorganismo afecta al ser humano, éste actúa como hospedador intermediario en un ciclo vital que normalmente tiene lugar en otros animales como los ovinos, porcinos, bovinos, y caprinos, entre otros. Debido a esta característica, la infección por E. granulosus se relaciona en forma directa con la cría de ganado, y es una de las enfermedades zoonóticas más frecuentes en Argentina. Durante 2010, Argentina reportó 385 casos de hidatidosis notificados al Sistema Nacional de Vigilancia de la Salud. Se calcula que el $30 \%$ del territorio de Argentina es asiento del ciclo zoonótico del E. granulosus, siendo las regiones afectadas el área de la Pampa Húmeda (Córdoba, sur de Santa Fe y Buenos Aires), el área Patagónica (Chubut, Santa Cruz, Tierra del Fuego, Neuquén y Río Negro), el área Mesopotámica (sur de Corrientes y el norte de Entre Ríos), el área Mediterránea (noreste de Córdoba, La Rioja, norte de San Luis y San Juan, sur de Catamarca y Santiago del Estero), el área Cuyana (Mendoza y el oeste de San Juan) y el área de Alta Montaña (Tucumán, Salta, Jujuy y

\footnotetext{
a Carrera de Medicina, Instituto Universitario Hospital Italiano de Buenos Aires. maria.ligo@hospitalitaliano.org.ar, morena.montalvo@hospitalitaliano.org.ar

b Servicio de Medicina Familiar, Hospital SAMIC EI Calafate.

c Departamento de Medicina, Instituto Universitario Hospital Italiano.

d Centro de Estudios de Estados y Sociedad. mariavictoria.salgado@gmail.com
} 
noroeste de Catamarca) ${ }^{1}$

Los quistes hidatídicos suelen ubicarse en distintos órganos, siendo la infección más frecuente en el hígado ( 67 al $89 \%$ ) y el pulmón (10 al 15\%), aunque también puede afectar el riñón, el cerebro, el corazón, los huesos y los músculos. Debido a esto, la clínica suele ser heterogénea y depende de la localización del quiste. Los pacientes pueden permanecer asintomáticos por periodos prolongados, en especial aquellos con quistes de localización hepática, en quienes los síntomas más frecuentes son el dolor en el hipocondrio derecho, las náuseas, los vómitos, la presencia de una masa palpable y de dispepsia no explicada por otras causas ${ }^{1}$

El diagnóstico de la hidatidosis se realiza mediante los hallazgos clínicos, los datos epidemiológicos y los exámenes complementarios $^{2}$. Entre estos últimos, la ecografía es uno de los estudios más utilizados por su bajo costo y accesibilidad ${ }^{1}$, aunque también se utilizan otros tipo de estudios complementarios, como por ej., los serológicos.

\section{Resumen de la evidencia}

Del Carpio M, et al. Diagnosis of human hydatidosis. Predictive value of a rural ultrasonographic survey in an apparently healthy population. Medicina (B Aires). 2000;60(4):466-8

Este trabajo ${ }^{3}$ se propuso evaluar la sensibilidad, la especificidad y el valor predictivo del rastreo de la hidatidosis hepática con ecografía en una población rural. Para ello, durante 1997 y 1998 se evaluaron mediante ecografía a 1.054 niños de 7 a 14 años de edad, de la localidad de Ingeniero Jacobacci, Río Negro, Argentina, utilizándose para los estudios poblacionales un equipo no moderno y un operador no especializado en diagnóstico por imágenes. Todos los casos detectados fueron derivados a un centro de alta complejidad especializado en diagnóstico por imágenes para su reevaluación con ecografía, tomografía computada (TC) y radiografía, consideradas en su conjunto como prueba de oro o gold standard. Se seleccionó un grupo control constituido por tres niños negativos por ecografía por cada caso positivo. Este grupo fue también reexaminado con ecografía, TC y radiografía en situaciones de duda. Se derivaron 27 portadores con imágenes positivas y sin síntomas clínicos de hidatidosis, y 66 clasificados como no portadores, confirmándose al reexamen 24 de los diagnosticados como portadores y la totalidad de los clasificados como sanos. En función de los resultados se estimó una sensibilidad de la ecografía para el diagnóstico de esta enfermedad del $100 \%$, con una especificidad del $95,6 \%$.

Si bien el estudio analizó población asintomática y no sospechosa de hidatidosis por sus manifestaciones clínicas, aporta información valiosa sobre las características operativas de la ecografía como método diagnóstico al utilizarla en condiciones no ideales.

\section{MacPherson C N, et al. Portable ultrasound scanner versus serology in screening for hydatid cysts in a nomadic population. Lancet. 1987;2(8553):259-61}

En este estudio ${ }^{4}, 3.553$ personas nómadas de Turkana, una zona remota del noroeste de Kenia, fueron examinados en busca de quistes hidatídicos mediante un ecógrafo portátil y por serología. De ellos, 198 (5,6\%) mostraron tener quistes en el hígado o en la parte superior del abdomen. En el grupo rastreado por ambas técnicas (2.644), $174(6,6 \%)$ casos de hidatidosis fueron detectados por ecografía y 76 (2,9\%) por serología. La ecografía dio resultados inmediatos y fue menos costosa, más aceptable y educativamente valiosa para la gente.
Yang Y R, et al. A correlative study of ultrasound with serology in an area in China co-endemic for human alveolar and cystic echinococcosis. Trop Med Int Health. 2007;12(5):637-46

Este estudio ${ }^{5}$ correlacionó la presencia de quistes hidatídicos hepáticos por ecografía con los resultados obtenidos por serología (ELISA e inmunotransferencia) en rastreos y seguimientos realizados entre 2001 y 2003 entre pacientes con hidatidosis confirmada por ecografía de la región de Ningxia, China. Entre 41 pacientes con casos confirmados por ecografía que accedieron a realizar la serología, el test de ELISA para Echinococcus granulosus resultó positivo en el $54 \%(22 / 41)$ de los casos; adicionalmente, otros 8 casos $(20 \%)$ reaccionaron de manera cruzada con el antígeno de Echinococcus multilocularis, mientras que en el $26 \%$ de los pacientes (11/41) ninguno de los test serológicos dio positivo.

\section{Stojkovic M, et al. Diagnosing and staging of cystic echinococcosis: how do CT and MRI perform in comparison to ultrasound? PLoS Negl Trop Dis. 2012;6(10):e1880}

El objetivo de este estudio ${ }^{6}$ fue determinar el rendimiento de la resonancia magnética nuclear (RMN) y la TC para el diagnóstico de quistes hidatídicos, en comparación con la ecografía. Se realizó un análisis retrospectivo de pacientes que presentaban quistes hidatídicos abdominales a quienes se le realizaron TC y/o RMN, como también ecografías, las cuales fueron analizadas por un mismo profesional con experiencia en el diagnóstico de hidatidosis. Se analizó la concordancia entre los estadios de los quistes determinados por la TC o la RMN y la ecografía, que se definió como prueba de referencia. La concordancia más allá del azar fue evaluada con el coeficiente kappa $(\kappa)$, considerando a los valores de 0,81 a 1,0 como muy buenos, los valores de 0,61 a 0,80 , buenos, y los de 0,41 a 0,60 , como moderados. Se analizaron 107 pacientes con 187 quistes; de ellos, los 187 quistes fueron evaluados por ecografía, 138 por TC y 125 por RMN. La concordancia más allá del azar con la ecografía en los quistes en estadio 1 a 4 de la clasificación de la OMS fue más baja para la $\mathrm{TC}$, con $\kappa$ que variaron entre 0,62 y 0,72 , en comparación con la RMN, con valores entre 0,83 y 1,0 . En cambio, en los quistes en estadío 5, la TC presentó mejor correlación con la ecografía que la $\mathrm{RMN}$ ( $\kappa=0,95$ vs. 0,65 respectivamente).

Los autores concluyeron que la ecografía continúa siento la piedra fundamental del diagnóstico, la estadificación y el seguimiento de los quistes hidatídicos. La RMN reproduce mejor que la TC las características definidas por la ecografía de estos quistes. Si no puediese realizarse una ecografía debido a la localización del quiste o a razones específicas del paciente, la RMN con uso de series ponderadas en T2 es preferible a la TC.

\section{Conclusiones y recomendaciones}

El estudio elegido para el diagnóstico temprano de hidatidosis hepática (la forma más común de hidatidosis), tanto en pacientes sintomáticos como en asintomáticos, debe ser, de manera ideal, sensible, específico, de bajo costo y sin efectos adversos. Los estudios aquí descritos continúan evidenciando que las características operativas de la ecografía igualan o superan a la de otros métodos de diagnóstico. En las investigaciones presentadas se constata que esta prueba cuenta además con la fortaleza de no necesitar la realización por operadores expertos ${ }^{3}$. Si sumamos a esta ventaja el bajo costo de este estudio, su portabilidad, y la ausencia de potenciales daños derivados de la radiación, concluimos que la ecografía continúa siendo el estudio de elección con el que iniciar la evaluación de pacientes con sospecha de hidatidosis hepática. Sin embargo, esto no significa que las pruebas serológicas u otros estudios de imágenes no sean útiles, sino que 
la decisión de utilizarlos debe basarse tanto en la clínica y la epidemiología del paciente como en los hallazgos observados en la ecografía.

Recibido el 12/04/2021 Aceptado el 05/07/2021 y Publicado el 24/07/2021.

\section{Referencias}

1. Argentina. Ministerio De Salud. Dirección de Epidemiología. Enfermedades infecciosas: Hidatidosis. Guía para el equipo de salud.; 2012. Available from: https://bancos.salud.gob.ar/sites/default/files/2018-10/0000000797cnt-2012-03-29_hidatidosis-guia-medica.pdf.

2. Brunetti E, Kern P, Vuitton DA, Writing Panel for the WHO-IWGE. Expert consensus for the diagnosis and treatment of cystic and alveolar echinococcosis in humans. Acta Trop. 2010;114(1):1-16. Available from: 10.1016/j.actatropica.2009.11.001.

3. Del-Carpio M, Moguilansky S, Costa M, et al. Diagnosis of human hydatidosis. Predictive value of a rural ultrasonographic survey in an apparently healthy population. Medicina (B Aires). 2000;60(4):466-468. Available from: https://www.medicinabuenosaires.com/demo/revistas/vol60-00/4/v60_ n_4_p466_468.pdf.

4. MacPherson CN, Romig T, Zeyhle E, et al. Portable ultrasound scanner versus serology in screening for hydatid cysts in a nomadic population Lancet. 1987;2(8553):259-261. Available from: 10.1016/s0140-6736(87)90839-7.

5. Yang YR, Craig PS, Ito A, et al. A correlative study of ultrasound with serology in an area in China co-endemic for human alveolar and cystic echinococcosis. Trop Med Int Health. 2007:12(5):637-646. Available from: 10.1111/j.1365-3156.2007.01834.x.

6. Stojkovic M, Rosenberger K, Kauczor HU, et al. Diagnosing and Staging of Cystic Echinococcosis: How Do CT and MRI Perform in Comparison to Ultrasound? PLoS Negl Trop Dis. 2012;6(10):e1880-e1880. Available from: 10.1371/journal.pntd.0001880;https://dx.doi.org/10.1371/journal.pntd. 0001880. 